\title{
HADAMARD MATRICES OF SMALL ORDER AND YANG CONJECTURE
}

\author{
DRAGOMIR Ž. ĐOKOVIĆ
}

\begin{abstract}
We show that 138 odd values of $n<10000$ for which a Hadamard matrix of order $4 n$ exists have been overlooked in the recent handbook of combinatorial designs. There are four additional odd $n=191,5767,7081,8249$ in that range for which Hadamard matrices of order $4 n$ exist. There is a unique equivalence class of near-normal sequences $N N(36)$, and the same is true for $N N(38)$ and $N N(40)$. This means that the Yang conjecture on the existence of near-normal sequences $N N(n)$ has been verified for all even $n \leq 40$, but it still remains open.
\end{abstract}

2000 Mathematics Subject Classification 05B20, 05B30

\section{INTRODUCTION}

Recall that a Hadamard matrix of order $m$ is a $\{ \pm 1\}$-matrix $A$ of size $m \times m$ such that $A A^{T}=m I_{m}$, where $T$ denotes the transpose and $I_{m}$ the identity matrix. Let us denote by $H M(m)$ the set of Hadamard matrices of order $m$. By abuse of language, we say that $H M(m)$ exist if $H M(m) \neq \emptyset$. If $m>2$ and $H M(m)$ exist, then $m$ is divisible by 4 . In the recent handbook [1, pp. 278-279] one finds a table of all odd integers $n<10000$ and exponents $t(2 \leq t \leq 8)$ for which it is known that $H M\left(2^{t} n\right)$ exist. The Hadamard conjecture asserts that we should always have $t=2$ (for $n>1$ ). We shall refer to the cases with $t \geq 3$ as bad cases. The good cases, i.e., those with $t=2$ are indicated in the table by a dot. The table has 5000 entries of which 1006 are bad. In this note we point out that 138 of these 1006 cases are in fact good. Additional 4 cases can be also eliminated, reducing the number of bad cases to 864 .

In the next section we recall a well known construction (see Proposition 2.1) of $H M(4 n)$ which uses Yang multiplication, T-sequences, orthogonal designs, and Williamson-type matrices. In section 3 we list 138 bad cases and invoke Proposition 2.1 to show that they should have been classified as good cases. We also mention four additional

Key words and phrases. Base sequences, normal and near-normal sequences, Tsequences, orthogonal designs, Williamson-type matrices, Yang conjecture. 
bad cases that we can eliminate. In section 4 we show that there exist near-normal sequences $N N(n)$ for $n=32,34,36,38$ and 40. Thus, despite of our efforts to find a counter-example, the Yang conjecture on near-normal sequences still remains open.

\section{Preliminaries}

Let us introduce the following notation for the sets of some important combinatorial objects (for their definitions see [1, 3, 4, 10]):

$\begin{array}{ll}G S(g) & \text { Golay sequences of length } g \\ T S(t) & \text { T-sequences of length } t \\ B S(r, s) & \text { Base sequences of lengths } r, s \\ N S(l) & \text { Normal sequences, as a subset of } B S(l+1, l) \\ N N(l) & \text { Near-normal sequences, as a subset of } B S(l+1, l) \\ O D(4 d) & \text { Orthogonal designs } O D(4 d ; d, d, d, d) \\ W T(w) & \text { Williamson-type matrices of order } w \\ B H W(4 h) & \text { Baumert-Hall-Welch arrays of order } 4 h\end{array}$

It is well known that there exist constructions (i.e., maps)

$$
\begin{aligned}
& G S(g) \rightarrow N S(g), \\
& G S(g) \rightarrow B S(g, 1), \\
& B S(r, s) \rightarrow T S(r+s), \\
& N S(l) \times B S(r, s) \rightarrow T S((2 l+1)(r+s)), \\
& N N(l) \times B S(r, s) \rightarrow T S((2 l+1)(r+s)), \\
& B H W(4 h) \times T S(t) \rightarrow O D(4 h t), \\
& O D(4 d) \times W T(w) \rightarrow H M(4 d w) .
\end{aligned}
$$

The first three constructions are elementary. They are given by

$$
\begin{aligned}
(A ; B) & \rightarrow(A,+; A,-; B ; B) \\
(A ; B) & \rightarrow(A ; B ;+;+) \\
(A ; B ; C ; D) & \rightarrow\left((A+B) / 2,0_{s} ;(A-B) / 2,0_{s} ;\right. \\
& \left.\quad 0_{r},(C+D) / 2 ; 0_{r},(C-D) / 2\right),
\end{aligned}
$$

where + and - stand for +1 and -1 , respectively, comma denotes the concatenation of sequences, and the symbol $0_{d}$ denotes the sequence of $d$ zeros. The arithmetic operations on sequences are performed component-wise. The constructions (2.4) and (2.5) are due to Yang [11]. For the "plug in" constructions (2.6) and (2.7) see [10] Theorems 3.10 and 3.8, respectively.

Recall that $B H W(4 h)$ exist for $h \in\{1,5,9\}$ (see [10]). An integer $g$ is a Golay number if $G S(g)$ exist. It is known that $2^{a} 10^{b} 26^{c}, a, b, c \geq 0$ 
integers, are Golay numbers. An odd integer $y=2 l+1$ is a Yang number if $N S(l)$ or $N N(l)$ exist. The following well known fact is an immediate consequence.

Proposition 2.1. If $y$ is a Yang number and $B H W(4 h), B S(r, s)$ and $W T(w)$ exist, then $H M(4 n)$ exist for $n=y h(r+s) w$.

Proof. We first apply the construction (2.4) or (2.5), whichever is appropriate, to obtain T-sequences of length $t=y(r+s)$. Next, we apply the construction (2.6) to obtain an $O D(4 h t)$. Finally, the construction (2.7) produces an $H M(4 n)$.

\section{AN update of THE List OF BAD CASES}

Let $\Delta$ be the following set of 138 odd integers $<10000$ :

$$
\begin{aligned}
& \{1397,2159,2413,2773,2921,3175,3953,4053,4083,4097, \\
& \text { 4181, 4227, 4307, 4389, 4439, 4453, 4479, 4495, 4499, 4589, } \\
& \text { 4633, 4659, 4747, 4765, 4859, 4921, 4981, 5017, 5165, 5199, } \\
& \text { 5201, 5207, 5211, 5259, 5317, 5359, 5363, 5379, 5383, 5411, } \\
& \text { 5461, 5545, 5567, 5597, 5619, 5667, 5709, 5825, 5875, 5913, } \\
& \text { 5915, 5965, 5969, 5979, 5989, 6001, 6059, 6129, 6341, 6351, } \\
& 6369,6495,6523,6605,6667,6693,6707,6731,6743,6755, \\
& 6805,6813,6893,6953,6985,6989,6995,7045,7093,7223, \\
& 7325,7373,7387,7413,7427,7439,7471,7493,7505,7571, \\
& 7613,7633,7709,7765,7913,7953,8033,8131,8155,8197, \\
& 8299,8327,8465,8477,8485,8503,8509,8579,8589,8633, \\
& 8655,8665,8743,8833,8899,8917,9005,9065,9071,9083, \\
& 9087,9093,9107,9169,9273,9325,9365,9407,9445,9485, \\
& 9515,9527,9549,9553,9827,9881,9959,9965\} .
\end{aligned}
$$

According to [1, pp 278-279] all these cases are bad, i.e., they all have $t \geq 3$.

We claim that the existence of $H M(4 n)$ for $n \in \Delta$ has been known for some time and that all these cases should have been classified as good. To prove this claim, it suffices to apply the above proposition. We used only the facts known for a few years prior to the publication of [1]. In more detail, we used the existence of $B S(l+1, l)$ for $l \leq 35$, $B S(2 l-1, l)$ for even $l \leq 36, B S\left(g_{1}, g_{2}\right)$ for $g_{1}$ and $g_{2}$ Golay numbers, and $N N(l)$ for even $l \leq 30$. For Williamson-type matrices $W T(w)$, we used the listing for odd $w<2000$ given in [10, Table A.1] with only two changes. Namely we used the fact that $W T(w)$ exist for $w=35$ 
and $w=127$, proven in [2] and recorded in [1, Table V.1.50, p. 277] as well.

Since the verification is of routine nature and tedious, we shall just give a few examples (see Table 1) and list the acceptable choices for the parameters $y, h,(r, s), w$. In some cases there are several such choices, which may give different constructions for $H M(4 n)$.

Since 2048 and 2600 are Golay numbers, it follows from (2.1) that $4097=2 \cdot 2048+1$ and $5201=2 \cdot 2600+1$ are Yang numbers. The $B S(r, s)$ that occur in Table 1 can be found in many places, e.g., [4, 10], except for the case $(r, s)=(34,33)$. In the exceptional case the base sequences were constructed in [9], see also below. Note that $B S(100,1)$, used for the case $n=7373$, exist because of (2.2).

Table 1: Parameters for the construction of $H M(4 n)$

\begin{tabular}{|c|c|c|c|c|c|c|c|c|c|}
\hline$n$ & $y$ & $h$ & $(r, s)$ & $w$ & $n$ & $y$ & $h$ & $(r, s)$ & $w$ \\
\hline 2773 & 59 & 1 & $(24,23)$ & 1 & 4495 & 31 & 5 & $(15,14)$ & 1 \\
\hline 3953 & 59 & 1 & $(34,33)$ & 1 & & 31 & 5 & $(1,0)$ & 29 \\
\hline \multirow[t]{2}{*}{4097} & 4097 & 1 & $(1,0)$ & 1 & & 31 & 1 & $(15,14)$ & 5 \\
\hline & 1 & 1 & $(2049,2048)$ & 1 & & 31 & 1 & $(3,2)$ & 29 \\
\hline \multirow[t]{15}{*}{4389} & 19 & 1 & $(17,16)$ & 7 & & 29 & 5 & $(16,15)$ & 1 \\
\hline & 19 & 1 & $(11,10)$ & 11 & & 29 & 5 & $(1,0)$ & 31 \\
\hline & 19 & 1 & $(6,5)$ & 21 & & 29 & 1 & $(3,2)$ & 31 \\
\hline & 19 & 1 & $(4,3)$ & 33 & & 29 & 1 & $(16,15)$ & 5 \\
\hline & 11 & 1 & $(29,28)$ & 7 & 5201 & 5201 & 1 & $(1,0)$ & 1 \\
\hline & 11 & 1 & $(11,10)$ & 19 & & 1 & 1 & $(2601,2600)$ & 1 \\
\hline & 11 & 1 & $(10,9)$ & 21 & 5875 & 25 & 5 & $(24,23)$ & 1 \\
\hline & 11 & 1 & $(4,3)$ & 57 & & 25 & 1 & $(24,23)$ & 5 \\
\hline & 11 & 1 & $(1,0)$ & 399 & & 5 & 1 & $(24,23)$ & 25 \\
\hline & 7 & 1 & $(29,28)$ & 11 & & 5 & 5 & $(24,23)$ & 5 \\
\hline & 7 & 1 & $(17,16)$ & 19 & & 1 & 5 & $(24,23)$ & 25 \\
\hline & 7 & 1 & $(10,9)$ & 33 & 5913 & 1 & 1 & $(41,40)$ & 73 \\
\hline & 7 & 1 & $(6,5)$ & 57 & 7373 & 1 & 1 & $(100,1)$ & 73 \\
\hline & 7 & 1 & $(1,0)$ & 627 & 9065 & 49 & 5 & $(19,18)$ & 1 \\
\hline & 1 & 1 & $(6,5)$ & 399 & & 49 & 5 & $(1,0)$ & 37 \\
\hline \multirow[t]{6}{*}{4453} & 1 & 1 & $(31,30)$ & 73 & & 49 & 1 & $(19,18)$ & 5 \\
\hline & & & & & & 49 & 1 & $(3,2)$ & 37 \\
\hline & & & & & & 37 & 5 & $(25,24)$ & 1 \\
\hline & & & & & & 37 & 5 & $(1,0)$ & 49 \\
\hline & & & & & & 37 & 1 & $(25,24)$ & 5 \\
\hline & & & & & & 37 & 1 & $(3,2)$ & 49 \\
\hline
\end{tabular}


There are four additional bad cases $n=191,5767,7081,8249$ that we can take care of, using new results. The construction of $H M(4 n)$ for $n=191$ appeared in [5]. For the remaining three cases we again apply Proposition 2.1. The acceptable choices for the parameters are $y=h=1,(r, s)=(37,36)$ and $w=79,97,113$, respectively. The main point is that we have shown that $N N(36)$ exist, and consequently $B S(37,36)$ exist (see the next section).

\section{The CURRent status of the YAng CONJECture}

In his paper [1] Yang said that "it is likely" that $N N(n)$ exist for all even integers $n>0$. This assertion has become known as "Yang conjecture", see e.g., [1, 4]. Note that, in our notation which is different from that of Yang, the set $N N(n)$ is empty for odd $n>1$. In our recent paper [6] we have introduced an equivalence relation for nearnormal sequences, to which we refer as $N N$-equivalence. This leads to a canonical form for $N N$-equivalence which is too technical to be given here. By using this canonical form we were able to enumerate the $N N$-equivalence classes in $N N(n)$ for even $n \leq 30$. Subsequently these exhaustive computations were extended to cover the cases of all even $n \leq 40$. For the cases $n=32$ and $n=34$ see our notes [7] and [8], respectively. After finding out that there is only one $N N$-equivalence class for $n=36$, we lost any hope that Yang conjecture may be true in general. However, to our great surprise, it turned out that there is again a single $N N$-equivalence class in $N N(38)$, and the same holds true for $N N(40)$. The computations in the last two cases were carried out on SHARCNET's machines running at $3.0 \mathrm{GHz}$. The CPU time for the case $n=40$ was about 1300 days.

Here we give examples of $N N(n)$ for $n=32,34,36,38,40$ in our encoded form:

$$
\begin{aligned}
& n=32 \quad: \quad[07651732153537650,1262758654155332], \\
& n=34 \quad: \quad[076417646512321462,16738541372344337], \\
& n=36 \quad: \quad[0764841234846532153,165154775335162126], \\
& n=38 \quad: \quad[07641237828515856281,1782612553714317675], \\
& n=40 \quad: \quad[058214351717346462170,11868533752571536124] .
\end{aligned}
$$

For the reader's convenience we also give an example of $B S(34,33)$ :

$$
\text { [07651732153537650, 1262758654155332]. }
$$

The encoding scheme is explained in our papers [3, 4, 6]. 


\section{ACKNOWLEDGMents}

The author is grateful to NSERC for the continuing support of his research. Part of this work was made possible by the facilities of the Shared Hierarchical Academic Research Computing Network (SHARCNET:www.sharcnet.ca).

\section{REFERENCES}

[1] C.J. Colbourn and J.H. Dinitz, Editors, Handbook of Combinatorial Designs, 2nd edition, Chapman \& Hall, Boca Raton/London/New York, 2007.

[2] D.Ž. Đoković, Good matrices of orders 33, 35 and 127 exist, J. Comb. Math. Comb. Comput. 14 (1993), 145-152.

[3] _ Base sequences, complementary ternary sequences, and orthogonal designs, J. Combinatorial Designs 4 (1996), 339-351.

[4] _ Aperiodic complementary quadruples of binary sequences, JCMCC 27 (1998), 3-31. Correction: ibid 30 (1999), p. 254.

[5] _ Hadamard matrices of order 764 exist, Combinatorica 28 (4) (2008), 487-489.

[6] Classification of near-normal sequences, Discrete Mathematics, Algorithms and Applications, 1, No. 3 (2009), 389-399. Available as a preprint on arXiv:0903.4390v2 [math.CO] 1 Sep 2009.

[7] _ Some new near-normal sequences, arXiv:0907.31290v1 [math.CO] 17 Jul 2009.

[8] _ A new Yang number and consequences, Des. Codes Cryptogr. (to appear).

[9] S. Kounias and K. Sotirakoglu, Construction of orthogonal sequences, Proc. 14-th Greek Stat. Conf. 2001, 229-236 (in Greek).

[10] J. Seberry and M. Yamada, Hadamard matrices, sequences and block designs, in "Contemporary Design Theory, A Collection of Surveys", J.H. Dinitz and D.R. Stinson, Eds., J. Wiley, New York, 1992.

[11] C. H. Yang, On composition of four-symbol $\delta$-codes and Hadamard matrices, Proc. Amer. Math. Soc. 107 (1989), 763-776.

Department of Pure Mathematics, University of Waterloo, WaterLoo, Ontario, N2L 3G1, Canada

E-mail address: djokovic@uwaterloo.ca 